Ringe übereinstimmen. Dieses Gebilde entspricht wohl einem kieselsäurehaltigen $\mathrm{Häutchen,}$ das sich gegen das Ende der Eindampfung aus der geringen Menge noch vorhandener Flüssigkeit bildete, die auf kapillarem Wege noch kurze Ausstrahlungen in die bereits an der trockenen Schalenwandung abgelagerte Substanz zu entsenden vermochte.

Die vom Verfasser hiermit beendeten Betrachtungen über die "Hysteresis" humoser wässeriger $B$ od e n a f $s \mathrm{ch}$ w $m$ mungen, welche die Fortsetzung der in dieser Zeitschrift bereits .früher erschienenen Abhandlung über „Hysteresis “ wässeriger Lösungen hum os er $\mathrm{B}$ ö d e $\mathrm{n}$ bilden sollen, wären aber unvollkommen, wenn nicht beigefügt würde, daß nach den angestellten Beobachtungen außer chemischen und physikalischenVorgängen auch bi olog is che Einflüsse an dem Zustandekommen der $\mathrm{Hy}-$ steresiswirkungen beteiligt gedacht werden können. Denn es siedelte sich nicht nur auf der Oberfläche der Versuchsflüssigkeiten fast regelmäßig eine "Kahnhaut" an, sondern es besetzten sich auch die Innenwandungen der Aufbewahrungsgefäße mit üppigen Kulturen niederer pflanzlicher Lebewesen. Die Art des Auftretens der niederen Flora zeigte einige. bemerkenswerte Eigentümlichkeiten. Der Belag an den Wandungen war entweder ein regellos gleichmäßiger oder es fand das Wachstum in senkrechter Zeilenform mit gleichmäßigen Zwischenrä̈umen von oben nach unten statt. In kurzer Entfernung vom Absatz der humosen Teilchen am Boden der Aufbewahrungsgefäbe machte das Wachstum der niederen Flora jedoch stets Halt, was mit der Anwesenheit saurer Bestandteile in der humosen Masse zusammenhängen dürfte.

\title{
Zur Frage der Heißvulkanisation des Kautschuks.
}

Bemerkungen zu den diesbezüglichen Veröffentlichungen von C. Harries.

Von F. Kirch h of (Wimpassing).

(Fingegangen am 6. Dezember 1919.)

Im Kapitel 7 seines ausgezeichneten Buches „Untersuchungen über die natürlichen und künstlichen Kautschukarten" "1) bespricht $\mathrm{C}$. Harries die Heißvulkanisation des Kautschuks. Auf Grund seiner Untersuchungen, welche $\mathrm{C}$. Harries im Jahre 1916 ausführte und veröffentlichte $\left.{ }^{2}\right)$ kam er zur Auffassung der Vulkanisation als eines physikalisch-chemischen Vorganges, der darin besteht, "daß der Naturkautschuk, als die metastabile Form, durch die Vulkanisation in eine andere, dichtere, träger reagierende Form, das Vulkanisat, umgewandelt wird".

Da Verfasser bereits 1914 ganz ăhnliche Ansichten vertrat ${ }^{3}$ ), C. $\mathrm{H}$ a rries aber weder in seinen Publikationen, noch in seinem zitierten Buch der Ansichten des Verfassers Erwähnung tut, so sieht sich dieser veranlaßt, auf die Priorität seiner diesbezüglichen Anschauungen besonders hinzuweisen.

Verfasser war bei der Interpretierung seiner damaligen Versuchsergebnisse im Banne der epochalen Untersuchungen C. $\mathrm{H}$ arries und legte daher ersteren die Olktadiënformel des Kautschuks zu Grunde. Da sich diese Auffassung als "zu eng" erwies, so wäre die

1) Verlag von J. Springer (Berlin 1919).

2) Ber. d. Deutsch. chem. Ges. 49, 1196 (1916), sowie Koll.-Zeitschr. 19, 1 (1916).

9) Koll.-Zeitschr. 14, 35 (1914) u. 15, 126 (1914). chemische Interpretation des Vulkanisationsvorganges entsprechend abzuändern ${ }^{4}$ ).

Verfasser hatte auf Grund seiner Untersuchungen "Zur Kenntnis der Additionsreaktionen des Kautschuks " ${ }^{5}$ )Vergleiche über die Aehnlichkeit des Vulkanisationsvorganges mit ersteren angestellt und kam damals zu folgendem Schlui : „Der rasche Absturz der Viskosität nach dem Hinzufügen eines reaktionsfähigen Stoffes (zu den Kautschuklösungen) ist nun in Analogie zur gleichen Erscheinung, die beim Erhitzen von Kautschuklösungen eintritt, auf eine Depolymerisation des Kautschuks zurückzuführen. Erst nach erfolgter Depolymerisation ist der Kautschuk zu Additionsreaktionen befähigt und in Lösungen verhält er sich dann wie ein Molekel mit zwei Doppelbindungen, da hier die Depolymerisation (nahezu) quantitativ verläuft. Bei den Reaktionen (des Kautschuks) im festen Zustand kommt es dagegen zur Bildung eines Gleichgewichtes, welches durch die Tendenz zur Verkettung der einzelnen Oktadiënringe herbeigeführt, resp. nach der Seite der Repolymerisation

4) Auf die Untersuchungen von W. C. Schmitz, Gummi - Ztg. 34, 167 und 1937 (1919), sowie die daraus gezogenen Folgerungen wird weiter unten zurückgekommen.

5) Ber. d. Deutsch. chem. Ges. 49, 1196 (1916), sowie Koll.-Zeitschr. 19, 1 (1916). 
verschoben wird. Da hierbei mehrere Faktoren, wie z. B. ursprünglicher Grad der Polymerisation, Stärke des depolymerisierenden Agens, Temperatur usw. in Frage kommen, so ist es klar, $\mathrm{da} \beta$ es hier zur Bildung von Verbindungen kommen kann, welche keine einfachen stöchiometrischen Verhältnisse mehr aufweisen. Derartige Reaktionen wurden öfter als „Adsorptionsvorgänge" bezeichnet.
Zufolge dieser Erscheinungen ist anzunehmen, daß (auch) bei der (Heib)-Vulkanisation ein Uebergang des durch weniger feste Bindungen bedingten Zustandes der Polymerisation, in stabile kondensierte Systeme stattfindet, analog wie dies durch andere Stoffe, z. B. $\mathrm{S}_{2} \mathrm{Cl}_{2}$, bewirkt wird. Schematisch kann dieser Vorgang wie folgt dargestellt werden:

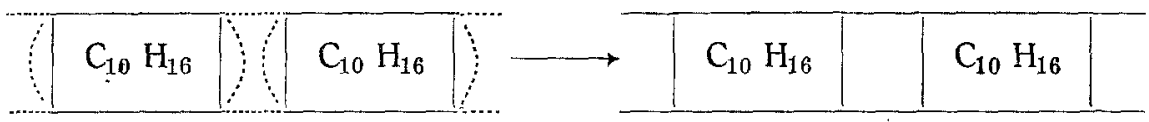

Polymerisiert

Auf Grund der damaligen Auffassung des Kautschuks als Dimethylzyklooktadiën versuchte ich nun eine strukturchemische Erklärung des Vorganges zu geben.

"Strukturchemisch gesprochen kommt es aber hierbei zurBildung von Tetramethylenringen, welche unbedingt stabilere Systeme als die Doppelbindungen darstellen. Immerhin kommt dem Tetramethylenring noch eine relativ hohe Reaktionsfähigkeit zu, was ja bei der Vulkanisation selbst, ferner bei der Oxydation und Ozonisation (sowie anderen Additionsreaktionen) des vulkanisierten Kautschuks zum Ausdruck kommt. GemäB dieser Reaktionsfähigkeit wird ja bei der Vulkanisation selbst ein Teil des Schwefels chemisch gebunden. Inwieweit diese beiden Vorgänge, Kondensation zum Tetramethylenring und Bindung des Schwefels voneinander abhängen, bleibt ferneren Untersuchungen überlassen ${ }^{6}$ ). Tatsache ist, daf der (vulkanisierte) Kautschuk von einem gewissen Grad der Schwefelung an, ein Optimum der physikalischen Eigenschaften erreicht hat. Eine weitere Schwefelbindung ist unbedingt als eine Reaktion mit dem Tetramethylenring aufzufassen."

Zum Schlusse der zitierten Abhandlung schrieb ich noch: „Die durch die Vulkanisationswärme bewirkte Depolymerisation wird die Ursache für die größere Reaktionsfähigkeit des Kolloids. In diesem Zustand wirkt nun der gleichfalls reaktionsfähiger gewordene Schwefel kondensierend auf die Oktadiënringe ein. Gleichzeitig findet Bindung eines Teiles des Schwefels statt. Letztere ist aber eher als eine notwendige Begleiterscheinung, als die eigentliche Ursache des $Z$ ustandes, vulkanisiert'auf $z$ ufassen. Als diese kommt vielmehr die konden-

6) Desgleichen die Frage, inwieweit Vulkanisationsbeschleuniger die beiden Vorgänge beeinflussen. sierende (katalytische) Wirkung in Betracht".

Es wurde also hier bereits klar zum Ausdruck gebracht, daß der eigentlichen chemischen S-Bindung (von C. Harries Nachvulkanisation genannt) ein rein physikalisch-chemischer Vorgang vorangeht bzw. mit ihm gleichzeitig verläuft, welcher blob durch die Gegenwart des Schwefels katalytisch beschleunigt wird.

Es müBte demnach, wie dies auch $C$. Harries ausgesprochen hat, eine HeiBvulkanisation auch durch andere Stoffe als Schwefel bewirkt werden können ${ }^{7}$ ).

Nach C. Harries ist der Uebergang der metastabilen Form des Kautschuks in die stabile von einer chemischen Aenderung der Eigenschaften begleitet. Die.Hydrochloride der beiden Formen sind nämlich nicht identisch. Das Hydrochlorid des gefällten und des umgefällten Naturkautschuks wird von Chloroform gelöst, dasjenige des entschwefelten Vulkanisats ist darin vollständig unlöslich. Die elementare chemische Zusammensetzung beider Hydrochloride ist die gleiche, ihre Zersetzungspunkte sind aber verschieden. In strukturchemischer Hinsicht ist bei beiden Formen ein Unterschied insoweit nicht zu erkennen, als bei der Oxyauftreten; indessen wird die stabile Form (Vulkanisat) schwerer von Ozon angegriffen. Nach C. Harries nimmt der Naturkautschuk beim Uebergang in das Vulkanisat wahrscheinlich eine andere Molekulargröße an.

Es wäre hier noch eine Erscheinung zu beachten, die in der Gummitechnik schon lange bekannt ist und auf die auch C. Harries (loc. cit.) hingewiesen hat.

7) Siehe Koll.-Zeitschr. 23, 25 (1918), sowie Ref. Gummi-Ztg. 33, 666 (1919). dation mit Ozon die gleichen Spaltungsprodukte 
Das mit Azeton erschöpfend extrahierte Primärvulkanisat gibt nach neuerlichem Vermischen mit Schwefel bei darauffolgender Vulkanisation keine technisch brauchbaren Vulkanisate, sondern graue bis schwarze, weiche, blasige Massen, auch wenn man die durch die Azetonextraktion entfernten Kautschukharze wieder hinzugesetzt hat. Das Kautschukmolekel hat eben anscheinend doch eine strukturelle Aenderung erfahren, da eine nachträgliche Einwirkung des Schwefels zu Nebenreaktionen (Abspaltung von $\mathrm{H}_{2} \mathrm{~S}$ ) führt und keine normalen Vulkanisate mehr ergibt $^{8}$ ).

Phänomenologisch und wahrscheinlich auch chemisch hat die Primärvulkanisation grobe Aehnlichkeit mit der Hitzekoagulation des $\mathrm{Ei}$ weiBes. Es ist nicht ausgeschlossen, daB auch letztere auf die kondensierende. Wirkung des Schwefels zurückzuführen sein dürfte.

$\mathrm{DaB}$ aber dem Schwefel nicht die alleinige Wirkung bei der Vulkanisation zukommt, konnte auch experimentell bestätigt werden.

Eine Mischung aus 100 Teilen Rohgummi (Crêpe hell), 60 Teilen Kreide, 1,5 Teilen Schwefel, 1 Teil organischen Beschleuniger (schwefelfrei) und 7 Teilen Bleiglätte wurde 40 Minuten bei 3 Atm. vulkanisiert. Das Vulkanisat zeigte sich sowohl dem Aussehen als seinem Verhalten nach bei der mechanischen Prüfung als vollständig ausvulkanisiert, während eine ganz analoge Mischung, welche aber kein $\mathrm{PbO}$, oder an Stelle dessen $\mathrm{MgO}$ oder eine größere Menge von organischem Beschleuniger enthielt, auch nach 60 Minuten Heizung noch typisch untervulkanisiert war.

$\mathrm{Da}$ das $\mathrm{PbO}$-haltige Vulkanisat schwarz war, sich also reichliche Mengen $\mathrm{PbS}$ gebildet hatten, so konnte nur sehr wenig Schwefel an den Kautschuk gebunden sein. Die Analyse ergab 0,55 Proz. gebundenen Schwefel.

Dem $\mathrm{PbO}$ scheint demnach in Verbindung mit künstlichen oder natürlichen organischen Beschleunigern eine spezifische Wirkung bei

8) Die Ursache für dieses Verhalten dürfte die folgende sein: Das durch die Primärvulkanisation bereits polymerisierte $\mathrm{bzw}$. kondensierte Primärvulkanisat wird bei neuerlicher Vulkanisation nicht mehr depolymerisiert (wie es ja auch unlöslich geworden ist) und daher ist es $z \mathfrak{u}$ neuerlicher weiterer Polymerisation bzw. Kondensation nicht mehr befähigt. Dieses Verhalten trifft aber nicht für alle Kautschuksorten $z u$, sondern schein't auf die hochwertigen Kautschuke beschränkt zu sein. - Minderwertige Sorten liefern bei der vorerwähnten Behandlung ganz brauchbare Vulkanisate. Auch beruht auf derselben ein Verfahrenr, um klebrige Rohgummis (Flakes) in höherwertige Produkte $\mathrm{zu}$ verwandeln. der Heißvulkanisation zuzukommen, worauf ja auch die Untersuchungen von Ostromisslensky und Bunschoten (loc. cit. Anm. 7) hindeuten.

Auch die nichtvulkanisierten $\mathrm{PbO}$-haltigen Mischungen gaben in Kautschukquellungsmitteln keine vollkommene Quellung (Lösung), so daß bereits eine Polymerisation beim Walzen stattgefunden haben mufte.

Was nun die chemische Interpretation des Vulkanisationsvorganges betrifft, so ist die frühere Ansicht des Verfassers, daß es dabei zur Bildung von Tetramethylenringen käme, nicht mehr aufrecht zu erhalten.

$\mathrm{Da}$ den Versuchsergebnissen Schmitz (loc. cit.) über die Bromierung von vulkanisiertem Kautschuk nicht vorgegriffen werden soll, so seien hier blob einige Ansichten, die auf Analogieschlüssen beruhen, ausgesprochen.

Schmitz fand bekanntlich (loc. cit.), daß depolymerisierter Kautschuk pro Molekül $\mathrm{C}_{20} \mathrm{H}_{32}$ nicht 8 , sondern 10 Atome Brom addiert, von denen 2 Atome leicht als $\mathrm{HBr}$ abspaltbar sind. Die Anlagerung der locker gebundenen Bromatome findet an endständigen $\mathrm{CH}_{2}$-Gruppen statt, welche bei der Depolymerisation des $\mathrm{C}_{20} \mathrm{H}_{32^{-}}$(oder eines größeren) Ringmolekels gebildet werden.

Die $\mathrm{HBr}$-Abspaltung geht möglicherweise unter Verkettung zweier Molekeln $\mathrm{C}_{20} \mathrm{H}_{30} \mathrm{Br}_{8}$ vor sich, da nach derselben keine neuen Doppelbindungen vorhanden sind, während zufolge der Unlöslichkeit des neuen Bromids in Tetrachlorkohlenstoff das Molekulargewicht desselben eir höheres als das des gewöhnlichen Tetrabromids sein dürfte. Bei dieser Verkettung könnten sich demnach Tetramethylenringe bilden :
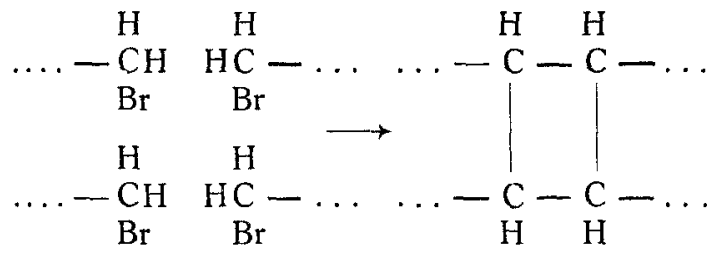

$\mathrm{C}_{20} \mathrm{H}_{32} \mathrm{Br}_{10} \quad \mathrm{C}_{20} \mathrm{H}_{32} \mathrm{Br}_{10} \quad\left(\mathrm{C}_{20} \mathrm{H}_{30} \mathrm{Br}_{8}\right)_{2}$

Analoge Vorgänge, wie sie hier bei der Bromierung stattfinden, scheinen nun auch bei anderen Additionsreaktionen des Kautschuks vor sich zu gehen. Hier sei nur auf die Erscheinungen der Kalt- und der Heißvulkanisation eingegangen. Hin richsen und Kindscher ${ }^{9}$ ) haben seinerzeit auf die Bildung einer Ver-

9) W. Hinrichsen und E. Kindscher, Koll.Zeitschr. 6, 202 (1910). 
bindung der Formel $\left(\mathrm{C}_{10} \mathrm{H}_{16}\right)_{2} \mathrm{~S}_{2} \mathrm{Cl}_{2}$ hingewiesen. $\mathrm{Da}$ der Bildung dieser Verbindung eine Depolymerisation des Kautschuks, mithin Bildung endständiger $\mathrm{CH}_{2}$-Gruppen vorangeht, so werden wir nicht fehlgehen, auch in ihr eine Anlagerung des $\mathrm{S}_{2} \mathrm{Cl}_{2}$ an die freien Bindungen der $\mathrm{CH}_{2}-$ Gruppen anzunehmen, etwa nach Formel I oder II, wobei II den Vorzug verdient, da sie eine Verkettung der Einzelmolekel (Vulkanisation) zum Ausdruck bringt:

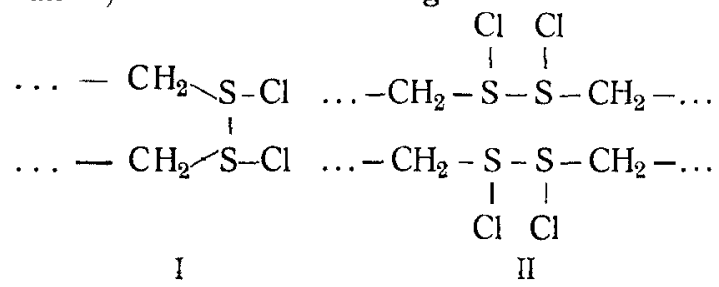

$\mathrm{Da}$ durch Behandlung mit alkoholischer Kalilauge das Chlor praktisch vollständig aus der Verbindung entfernt werden kann, so scheint es in diesem Fall wieder zu einer ähnlichen Konfiguration wie beim Bromid $\mathrm{C}_{20} \mathrm{H}_{30} \mathrm{Br}_{8}$ zu kommen, vorausgesetzt, daß dabei tatsächlich $\mathrm{HCl}$ - Abspaltung stattfindet :

$$
\ldots-\underset{\mathrm{I}}{\mathrm{C}}-\mathrm{S}-\mathrm{S}-\underset{\mathrm{C}}{\mathrm{C}}-\mathrm{C}-\mathrm{C}-\mathrm{S}-\underset{\mathrm{C}}{\mathrm{C}}-\ldots
$$

Die „Doppelbindungen“ des Kautschuks kommen demnach bei der Verbindung III anscheinend gar nicht zur. Absättigung.

Ganz Aehnliches scheint nun auch bei der Heißvulkanisation, wenigstens bei den niedrigeren Temperaturen, der Fall zu sein.

Der Vulkanisation geht, wie vielfach nachgewiesen wurde, eine Depolymerisation der Ringmolekel voraus. Es ist naheliegend, auch hier wieder als erste Stufe der Vulkanisation eine Reaktion zwischen Schwefel und den freien Bindungen der $\mathrm{CH}_{2}$-Gruppen unter Verkettung zweier oder mehrerer $\mathrm{C}_{20} \mathrm{H}_{32^{-}}$(oder $\mathrm{C}_{25} \mathrm{H}_{40^{-}}$) Molekeln anzunehmen, etwa in folgender Form (III und IV):

$$
\begin{gathered}
\ldots-\mathrm{CH}_{2}-\mathrm{S}-\mathrm{S}-\mathrm{CH}_{2}-\ldots \\
\ldots-\mathrm{CH}_{2}-\mathrm{S}-\mathrm{S}-\mathrm{CH}_{2}-\ldots \\
\left(\mathrm{C}_{25} \mathrm{H}_{40} \mathrm{~S}_{2}\right)_{2} \\
\text { III } \\
\ldots-\mathrm{CH}_{2} \backslash \mathrm{S}=\mathrm{S}-\mathrm{CH}_{2}-\ldots \\
\left(\mathrm{CH}_{2}-\ldots\right. \\
\left(\mathrm{C}_{25} \mathrm{H}_{40} \mathrm{~S}\right)_{2} \\
\text { IV }
\end{gathered}
$$

Der Formel IV entspricht ein Schwefelgehalt von 8,6 Prozent. Die Existenz einer derartigen Verbindung liebe sich vielleicht aus den Versuchsergebnissen von Spence und Young ${ }^{10}$ ) folgern. Sie erhielten bei ihren Versuchen mit 10 Proz. Vulkanisationsschwefel Werte von gebundenem Schwefel zwischen 8,3 und 8,46 Proz.

Nun wäre es aber auch denkbar, daß der Schwefel, welcher bis zu seinem Siedepunkt $\left(448^{\circ} \mathrm{C}\right)$ das Molekel $\mathrm{S}_{8}$ besitzt, bereits als solches (mit Hilfe von Partialvalenzen) mit den freien Bindungen der $\mathrm{CH}_{0}$-Gruppen in Reaktion träte und daB erst allmählich eine Dissoziation in $\mathrm{S}_{2}$-Molekel stattfände. In ersterer Bindungsform müfte der Schwefel auch durch physikalische Lösungsmittel extrahierbar sein. Diese Form des Schwefels liegt wahrscheinlich bei den Primärvulkanisaten (s. Harries loc. cit.) vor und ist möglicherweise identisch mit dem "adsorbierten" Schwefel. Aber auch der in obiger Formel als ,chemisch" gebundener auftretende Schwefel scheint bei der Behandlung mit chemischen Lösungsmitteln für Schwefel (also bei der Regeneration) vollständig entfernbar zu sein, wobei eine teilweise Rückbildung der $\mathrm{C}_{20} \mathrm{H}_{32^{-}}$bzw. $\mathrm{C}_{25} \mathrm{H}_{40^{-}}$-Ringe erfolgen dürfte, da das so entschwefelte Produkt wieder rohgummiähnliche Eigenschaften angenommen hat.

Erst bei viel höherer Temperatur (über $130^{\circ}$ ) und höherer Schwefelkonzentration (über 10 Prozent), welche Vërhältnisse bei der Hartgummivulkanisation vorliegen, addiert das Kautschukmolekel auch an seine Doppelbindungen beträchtliche Mengen Schwefel ${ }^{11}$ ). Dieser ist durch die gewöhnlìchen physikalischen und chemischen Lösungsmittel des Schwefels und ohne tieferen Eingriff in das Kautschukmolekel nicht mehr aus diesem $z u$ entfernen. Er liegt wahrscheinlich als Einzelatom an die Doppelbindungen angelagert vor.

10) D. Spence und J. Young, Koll.-Zeitschr. 11, 28 (1912). Der Gesamt - S - Gehalt ihrer Mischungen war nach 20 stündiger Vulkanisation von 9,9 auf 8,46 , bzw. von 10,06 auf 8,3 Prozent zurückgegangen. Diese Schwefeldifferenzen kann man sich nur durch Verflüchtigung von freiem Schwefel erklären. Ganz analog sind die Differenzen bei den Versuchen mit 37 Proz. $\mathrm{S}$ auf Verflüchtigung zurückzuführen. Siehe weiter Anm. 12.

11) Eine lebhafte Reaktion zwischen Schwefel und Doppelbindungen setzt erst über $160^{\circ} \mathrm{C}$ ein (Faktisbildung). Diese Temperatur fällt mit der Bildung der dunklen, zähflüssigen Modifikation des Schwelels zusammen. 
Es ist interessant, dab auf Grund dieser Annahmen das höchste Schwefelungsprodukt mehr als 32 Prozent $S$ enthalten müBte, was auch schon. Weber u. a. behauptet haben. Nimmt man als einfachstes Kautschukmolekel $\mathrm{C}_{20} \mathrm{H}_{32}$, oder, was nach den Untersuchungen $\mathrm{Harries}$ wahrscheinlicher ist, $\mathrm{C}_{25} \mathrm{H}_{40}$ an, so ergibt sich als schematische Strukturformel für das Höchstvulkanisat die folgende, worin die dicken Striche eine $\mathrm{C}_{5} \mathrm{H}_{8}$-Gruppe mit einer Doppelbindung andeuten sollen:

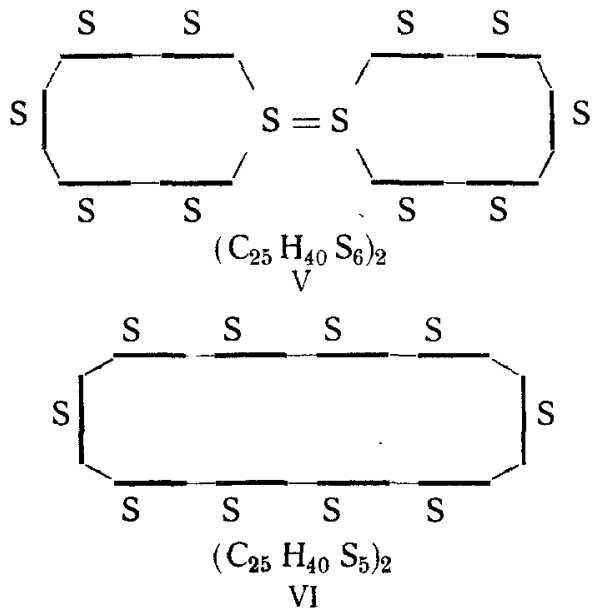

Für eine derartige Verbindung (V) berechnen sich 36,1 Prozent S, während die, durch die bloße Absättigung der Doppelbindungen gebildete Verbindung (VI) 32,1 Prozent verlangt. Tatsächlich scheint erstere in den zitierten Versuchen von Spence und Yo u ng vorzuliegen, welche nach 30 stündiger Heißvulkanisation (1450) mit 37 Prozent Mischungsschwefel, einen Gesamtschwefelgehalt des Vulkanisats von 35,8 Prozent fanden, während der gebundene Schwefel (nach der Extraktion mit Azeton) 31,97 Prozent betrug ${ }^{12}$ ).

Diese Betrachtungsweise erscheint nach Ansicht des Verf. geeignet, die alte Streitfrage, die sich hinsichtlich der Definition des Vulkanisationsschwefels ergab, zu klären. Man hat nach der dargelegten Auffassung zwei Arten von' ,Vulkanisationsschwefel“ zu unterscheiden:

12) Es ist auch hier wieder interessant zu konstatieren, daß der ursprüngliche $S$ - Gehalt von 37,4 Pro$z$ ent nach 20 stündiger Vulkanisation auf 35,8 Prozent zurückgegangen ist. Da in 20 Stunden sich demnach 37,4 $-35,8=1,6$ Ptozent $S$ verflüchtigt hatten, so müßte der "freie" Schwefel in weiteren 10 Stunden abermals abgenommen haben. Da dies nicht der Fall ist, so muß man die obigen 35,8 Prozent tatsächlich als "gebundenen" Schwefel ansehen, wovon 3,8 Prozent allerdings lockerer gebunden erscheinen.
Einen molekular $\left(S_{2}\right.$ oder $\left.S_{8}\right)$ an die endständigen $\mathrm{CH}_{2}$-Gruppen des aufgespaltenen $\mathrm{C}_{20} \mathrm{H}_{32}$ - bzw. $\mathrm{C}_{25} \mathrm{H}_{40}$-Ringes gebundenen, welcher die Kondensation (Vulkanisation) der depoly. merisierten Einzelmolekel bewirkt; dieser Teil des Vulkanisationsschwefels ist durch physikalische bzw. chemische Schwefellösungsmittel bei entsprechender Dauer und Temperatur der Behandlung wieder entfernbar, wodurch der Vulkanisationsprozeb teilweise wieder rückgängig gemacht wird (Regeneration). Der andere Teil des Vulkanisationsschwefels, der (höchstwahrscheinlich) atomistisch an die Doppelbindungen des Kautschukkohlenwasserstoffs angelagert ist und die Hornisierung (Hartgummibildung) bewirkt, kann durch die gewöhnlichen Extraktionsund Regenerationsmittel nicht mehr entfernt werden ${ }^{13}$ ). Da die erste Art des Vulkanisationsschwefels ziemlich labil an den Kautschuk gebunden erscheint, so erklärt sich nun vielleicht auch eine katalytische Wirkung des ersteren bei der Vulkanisation: Der Vulkanisationsschwefel erster Art bewirkt die Kondensation mehrerer Einzelmolekel. Infolge seiner lockeren Bindung an die $\mathrm{CH}_{2}$-Gruppen vermag er aber auch mit anderen in der Mischung anwesenden Komponenten z.B.mit organischen Beschleunigern zu reagieren, wodurch die kondensierten Systeme in polymerisierte übergehen, möglicherweise nach folgendem Schema [VII und VIII ${ }^{14}$ ):

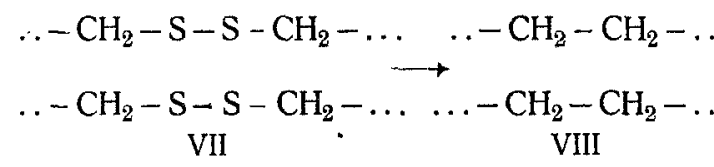

Dafs auch andere Stoffe in analoger Weise $\mathrm{zu}$ wirken vermögen, ist nach den oben erwähnten Versuchen von Bunschoten sowie des Verfassers als erwiesen $\mathrm{zu}$ betrachten ${ }^{15}$ ).

18) Die erste Art des Vulkanisationsschwefels käme bei der Primär-, die zweite bei der Nachvulkanisation gemäß der Harries'schen Auffassung in Betracht. Eine scharfe Grenze zwischen beiden läßt sich wohl praktisch überhaupt nicht ziehen, da eine minimale Schwefelbindung der zweiten Art, auch bei der Primärvulkanisation stets auftreten wird. Für gewöhnliche Verhältnisse können die von Harries angegebenen Bedingungen genügen.

14) Eine Abspaltung von $\mathrm{H}_{2} \mathrm{~S}$ in Analogie zur $\mathrm{HBr}$-Abspaltung bei den Bromverbindungen kommt bei den normalen Vulkanisations- und Regenerationstemperaturen nicht in Betracht, da die Affinität des Schwefels zum Wasserstoff weitaus geringer ist als diejenige des Broms.

15) Die Wirkung der Vulkazite und Akceleratoren scheint in erster Linie auf einer reaktionserhöhenden (dissoziierenden) Wirkung auf den Schwefel $z u$ beruhen. 


\section{Zusammenfassung.}

Verf. glaubt durch vorliegende Ausführungen dargetan $z \mathfrak{u}$ haben, daß er bereits vor Harries das physikalisch-chemische Prinzip der Heibvulkanisation erkannt und ausgesprochen hatte. Harries gebührt das unbestreitbare Verdienst, den Unterschied zwischen Primär- und Nachvulkanisation experimentell festgestellt $z u$ haben.

Zum Beweis, daß auch andere Stoffe als Schwefel eine vulkanisationsartige Wirkung auszuüben vermögen, wurden einige Versuche mit Bleiglätte in Verbindung mit wenig Schwefel und organischen Beschleunigern ausgeführt.
Die chemische Interpretation des Vulkanisationsvorganges mußte begreiflicherweise mancherlei Modifikationen erfahren, einerseits zufolge der Erweiterung unserer Erkenntnis von der Konstitution des Kautschuks durch Harries, anderseits durch die Untersuchungen Schmitz über das Verhalten von depolymerisierten Kautschuk bei der Bromierung.

In Analogie zu letzterem Vorgang wurde die Kalt- und speziell die Heißvulkanisation chemisch zu erklären versucht, wobei nochmals betont sei, daß hierdurch den in Aussicht gestellten experimentellen Ergebnissen W. C. Sch mitz in keinerWeise vorgegriffen werden soll.

\title{
Die elektrische Charakteristik der Farbstoffkolloide.
}

\author{
Von Rudolf Keller (Prag). \\ (Eingegangen am 15. Dezember 1919.)
}

(Schlukmitteilung.)

Kürzlich wurde an dieser Stelle ${ }^{1}$ ) mitgeteilt, daß die Angabe der Kolloidhandbücher, basische Farbstoffe wanderten an den Basenpol, an die Kathode, saure umgekehrt, unrichtig ist. Seit diese Versuche fortgesetzt wurden, hat sich auch herausgestellt, welche Tatsache dieser Litefaturangabe zugrunde liegt. Fast alle Lösungen kolloider Farbstoffe, insbesondere die Teerfarben, sind nicht einfache kolloid-disperse Systeme, sondern bestehen aus zwei Phasen, die je nach Löslichkeit, Verdünnungsgrad, Dispersionsmittel hervortreten, erstens der kolloiden Phase, für die die Gesetze der Kataphorese der Neutralkolloide vom Typus EiweiB, Zucker gelten, die also in Säuren und Alkalien umgekehrt wandern, zweitens der molekulardispersen Phase, oder eigentlich ionendispersen Phase, die vom elektrischen Strom wie ein anderes farbloses Ionendispersoid zersetzt wird, wobei natürlich ein gefärbtes Kation zur Kathode wandert: Da mit Vorliebe die Sulfosäuren der sauren Farbstoffe und einige sehr leicht lösliche gewöhnlich basisch genannte Farbstoffe untersucht worden sind, und zwar in verdünnten, stark dissoziierten Lösungen, so mußte der Irrtum entstehen, daß die gefärbten Kolloide anders wandern, als jene, die gewisse sichtbare Lichtstrahlen absorbieren. Mag es also praktisch zutreffen, daß sehr verdünntes Methylenblau in alkalischer Lösung kataphoretisch wandert - und nicht anaphoretisch wie meine kolloiden dicken Methylenblauschlieren - so gehört die Tatsache nicht in das Gebiet der Kolloidchemie

1) Koll.-Zeitschr. 25, 60 (1919). und mub scharf unterschieden werden von der eigentlichen Kolloidwanderung; es liegt ja im Wesen der Kolloide, daß infolge der Größe der Moleküle und infolge von Besonderheiten der Molekularoberfläche bei ihnen die Oberflächenkräfte einschlieblich der Ionenadsorption stärker hervortreten als die aus ihrem inneren chemischen Aufbau resultierenden Energien, die wieder das Verhalten der ionendispersen Verbindungen im Dispersionsmittel beherrschen.

Letzthin wurde erwähnt, daß meine Wanderungsversuche unter Laboratoriumsströmen nicht mit Kontrollversuchen nach der FließpapierStreifen-Methode von Fichter-Sahlbom übereinstimmten. Diese Unstimmigkeit hat sich inzwischen geklärt, und zwar durch den Versuch von H. Schmidt-Düsseldorf ${ }^{2}$ ). Dieser Autor hat Tropfen verdünnter Salzsäure auf Löschpapier fallen lassen und mit geeigneten Indikatoren, z. B. Kongorot, festgestellt, daß die Salzsäure gegenüber dem reinen Wasser in der kapillaren Ausbreitung zurückbleibt. Der Versuch zeigt also, daß sich die Lösung während des kapillaren Aufstiegs im Löschpapier derart durch Strömungsströme auflädt, daß - die Eintauchstelle als unten bezeichnet - die Anionen sich unten sammeln und die Kationen oben vorauseilen. Diese empirische Beobachtung selber findet sich schon in älteren histologischen Handbüchern nach Vorschriften aus dem Jahre 1888 erwähnt $^{3}$ ). Das Farbgemisch für Kernteilungen von $\mathrm{Ehrlich}, \mathrm{Bi}$ ondi, R. Heiden.

2) H. Sch m i dt, Koll.-Zeitschr. 24, 49 (1919).

3) Zeitschr. f. wissensch. Mikrosk. 5, 520 (1888). 